\title{
Simultaneous OH PLIF/Chemiluminescence and stereoscopic PIV measurements of combustion oscillation onset in turbulent swirling lean premixed flames
}

\author{
Yuki ATAGI*, Masayasu SHIMURA* and Mamoru TANAHASHI* \\ *Tokyo Institute of Technology, \\ 2-12-1 Ookayama, Meguro-ku, Tokyo 152-8550, Japan \\ E-mail: atagi.y.aa@m.titech.ac.jp
}

Received: 24 May 2020; Revised: 29 July 2020; Accepted: 17 September 2020

\begin{abstract}
To yield an effective control to impede the progress of combustion oscillation and to lead to the development of completely stable combustors, it is necessary to reveal the mechanism of the destabilization. For revealing the destabilization characteristics of combustion oscillation, methane-air turbulent lean premixed flames in a swirl-stabilized combustor were investigated by high-speed simultaneous measurements of stereoscopic particle image velocimetry (SPIV) arranged side by side, OH planar laser induced fluorescence (OH PLIF), $\mathrm{OH}$ chemiluminescence and pressure fluctuation. The transition process from stable state to unstable state of combustion oscillation is defined based on the root-mean-square $(\mathrm{rms})$ values of pressure fluctuation $p^{\prime}{ }_{\mathrm{rms}}$. Experimental condition was set as the swirl number of 1.14, equivalence ratio of 0.69 and total flow rate of 350 $\mathrm{L} / \mathrm{min}$ where the transition process is observed. In the transition process, magnitudes of fluctuating properties gradually gain. Pressure fluctuation phase-based analyses clarified that, as the transition process advances, intermittent large-scale vortical motion in the outside of sheer layer expands and approach the inlet, which has a close affinity with the growth of oscillation with making burnt regions involve unburnt regions. The transition process holds well for the Rayleigh criteria in that heat release fluctuates approximately the same phase of pressure fluctuation. On the other hand, in-flow velocity fluctuates in the antiphase of pressure fluctuation.
\end{abstract}

Keywords : Swirl-stabilized lean premixed flames, Combustion oscillation, Stereoscopic particle image velocimetry(SPIV), Planar laser induced fluorescence(PLIF), Chemiluminescence

\section{Introduction}

The importance of further research and development on combustors regarding the environmental issues is pointed out, especially in achieving higher thermal efficiency and reduction of nitrogen oxides emission. In the case of gas turbine swirl combustors which are utilized for thermal power generator and engines, an introduction of lean premixed flames is considered to be promising to suppress nitrogen oxides emissions drastically with improving thermal efficiency. However, lean premixed flames are fluctuation sensitive to provoke a thermoacoustic instability called combustion oscillation which will damage to the device severely. Therefore, combustion oscillation in the swirl-stabilized flame of gas turbine combustors is controversial for decades as the instability sets a complicated limit on safety operation (Lieuwen, et al., 2003). Lord Rayleigh stated the fundamental criteria for occurrence of the thermoacoustic instability (Rayleigh, 1878),

$$
\int_{\Omega} p^{\prime} q^{\prime} d V>L
$$

where $p^{\prime}$ and $q$ ' are fluctuations of pressure and heat release rate (HRR), $\Omega$ and $L$ indicate volume of the combustor and 
acoustic energy losses. The criteria states that the instability will occur under coupling between pressure and heat release fluctuations. The prediction and control of combustion oscillation has remained elusive and still been remarked as important scientific and technical challenges (Candel, et al., 2002). Therefore, the complex feedback process among fluctuations of HRR, pressure and velocity has been scrutinized for long time.

Investigations on the mechanism of sound generation in turbulent reactive mixing layer and the acoustic source components in Lighthill's acoustic analogy were conducted by using direct numerical simulation (DNS) (Li, et al., 2000). Thermoacoustic models were also considered and the interpretation for intrinsic acoustic modes was contrived (Bomberg, et al., 2014). However, there are recent progresses against thermoacoustic instabilities by numerical manners, Large Eddy Simulation (LES) which is a major tool to inquire combustion fields has not been sufficient yet (Poinsot, 2017).

The experimental strategies were also performed. The inlet temperature and equivalence ratio are revealed to be important factors for the destabilization (Huang, et al., 2004). Shanghogue et al. (2009) investigated anchoring effects on the combustion instabilities near the inlet with installing a bluff-body. The operation conditions in the real industrial combustors is maintained under the stable state; thus the combustors come to halt once combustion oscillation occur. However, several conditions which occur transition from stable state to unstable state are susceptible to any unpredicted disturbances such as velocity fluctuations or an abnormality of cooling systems. Shroll et al. (2012) looked into the stability characteristics by using dilution $\mathrm{CO}_{2}$ to control the flame temperature and reported the hysteresis features with respect to mode dependent transitions. Nair, et al. (2014) investigated an intermittency route of transition from combustion noise to combustion instability in the bluff-body equipped swirl-stabilized combustors and reveal that bluff-body-stabilized configuration has a hysteresis zone of instabilities. They also discussed that high-amplitude pressure oscillation will presage combustion instabilities. Renaud et al. (2015) studied the transition to stable state with changing the staging parameter in a swirl-stabilized turbulent spray combustor and noted the effects of Inner Recirculation zones (IRZ) and the helical vortex core called PVC which is distinctive to the swirling flows. As for the measurement methods for swirling flows, non-intrusive laser diagnostics has been recognized to be practical for the decades (Tanahashi, et al., 2017). Choi et al. (2005) investigated flame structure in relation with pressure fluctuation in a swirl stabilized combustor by using $\mathrm{OH}$ planar laser induced fluorescence (PLIF) and clarified that the large-scale flame structure change with beating frequency of pressure. The combustion field in a gas turbine model combustor was investigated by using laser Raman scattering, laser Doppler velocimetry (LDV) and planar laser induced fluorescence (PLIF) of $\mathrm{OH}$ and $\mathrm{CH}$ radicals (Weigand, et al., 2006). Shimura et al. (2009) investigated flow structures in combustion oscillation state in the swirl-stabilized combustor by using stereoscopic particle image velocimetry (SPIV) and have clarified characteristics of dynamics of large-scale vortex and recirculation zones in combustion oscillation state from velocity fields averaged in the phase of pressure in a swirl premixed combustor. Stöhr et al. (2009) conducted phase-based analyses against a helical vortex based on simultaneous measurements of SPIV and OH PLIF and clarified that the structure of reaction zone and velocity distribution temporally changes. Regarding the vortex formation, Steinberg, et al. (2013) clarified that the periodical vortex shedding in shear layers by combustion oscillation prevented a formation of helical vortex which is dominant structure in swirl-stabilized non-reaction flows. To clarify the instability in shear layer, linear stability analysis was conducted for axial forced swirl-stabilized flames (Oberleithner, et al., 2015). Flame-vortical interaction was visualized by Proper Orthogonal Decomposition (POD) strategy for the results of SPIV and OH PLIF in a gas turbine model combustor (Boxx, et al., 2010). The high-speed simultaneous measurements of PIV and OH PLIF are confirmed to be feasible also in the high-pressured gas turbine model combustor (Boxx, et al., 2015).

The objective of this study is to deepen understandings on the transition mechanism on the flow characteristics, flame structures and pressure fluctuation towards combustion oscillation, which will contribute to detection of onset of combustion oscillation and to development of robust and rapid control of combustion oscillation. In this study, simultaneous measurements based on SPIV and OH PLIF besides OH chemiluminescence and pressure measurement were performed within the transition process of turbulent swirling premixed flames to scrutinize how physical properties mutually interact with each other in the spatial distributions. The measurement area was set to be large enough to see both sides across the axis of mainstream direction and to recognize instantaneous differences such as asymmetrical movements. Therefore, measurement area of SPIV were arranged side by side with obtaining large area and sufficient spatial resolution. Fluctuating physical properties were organized corresponding to the magnitudes of pressure fluctuation and the transition process towards combustion oscillation was extracted. In the following sections, 
(a)



(b)

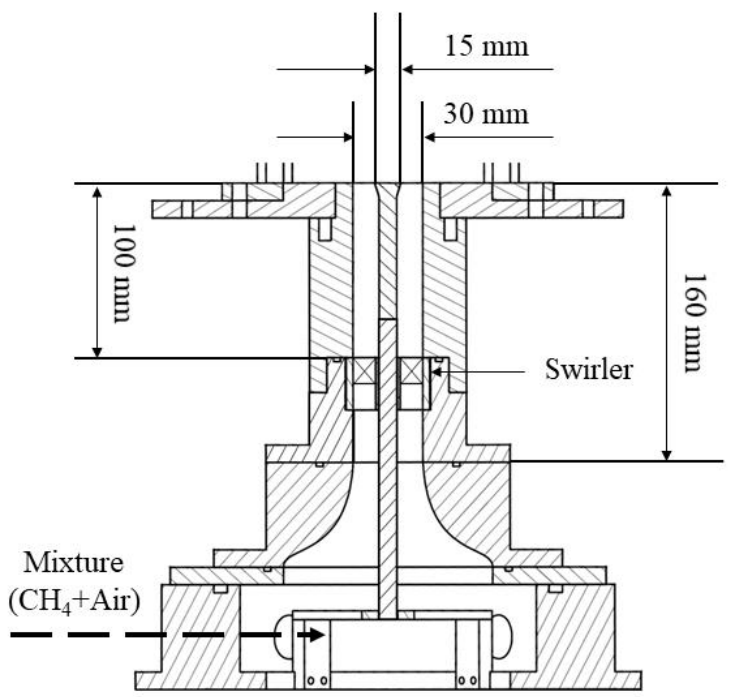

Fig. 1 (a) A schematic of a swirl combustor. The combustion chamber is rectangular of $120 \mathrm{~mm} \times 120 \mathrm{~mm}$ cross section and $1185 \mathrm{~mm}$ height. A pressure sensor is attached at $450 \mathrm{~mm}$ above the inlet of the combustion chamber. (b) Enlarged figure from a construction section to the inlet. Mixture gas of methane and air is led to the combustion chamber through ceramic balls, a construction section, a swirler and a straight metal tube of $30 \mathrm{~mm}$ diameter equipped with a centered bluff-body of $15 \mathrm{~mm}$ diameter.

the simultaneous measurement methods are described in Sec. 2, and the results in the transition process of a turbulent lean swirl-stabilized flame are shown and discussed in Sec. 3. The concluding remarks are addressed in Sec. 4.

\section{Apparatuses and conditions}

\subsection{Swirl-stabilized premixed combustor}

Figure 1(a) shows a schematic of a swirl-stabilized combustor used in this study. The combustor consists of a contraction section, swirler equipped inlet zone and a combustion chamber. The combustion chamber is rectangular of $120 \mathrm{~mm} \times 120 \mathrm{~mm}$ cross section and $1185 \mathrm{~mm}$ height. The acoustic modes of the combustor $f_{\mathrm{n} \times \mathrm{ny}, \mathrm{nz}}$ can be derived by

$$
f_{n_{x}, n_{y}, n_{z}}=\frac{c}{2} \sqrt{\left(\frac{2 n_{x}-1}{2 l_{x}}\right)^{2}+\left(\frac{n_{y}}{l_{y}}\right)^{2}+\left(\frac{n_{z}}{l_{z}}\right)^{2}}
$$

where $c, n_{i}$ and $l_{i}$ represent for the speed of sound, a mode and length of the combustor in $i$ direction $(i=x, y, z)$. The frequency of quarter-wave longitudinal acoustic mode is expected to be one of dominant modes (Aoki, et al., 2015), and that of the combustor is $72 \mathrm{~Hz}$ at about $300 \mathrm{~K}$. A pressure sensor is installed in the chamber at $450 \mathrm{~mm}$ above the inlet. On each side of the chamber right after the inlet, quartz glass windows are introduced to allow optical measurements. Figure 1(b) shows a sectional view of the combustor between a contraction section and a top of bluff-body. Mixture of methane and air gases, which are regulated in the upstream region by mass flow controllers, is led to the combustion chamber through a swirler and a straight metal tube of $30 \mathrm{~mm}$ diameter equipped with a centered bluff-body of $15 \mathrm{~mm}$ diameter. The swirler has 8 swirl vanes inclined 45 degrees from the central axis of the 


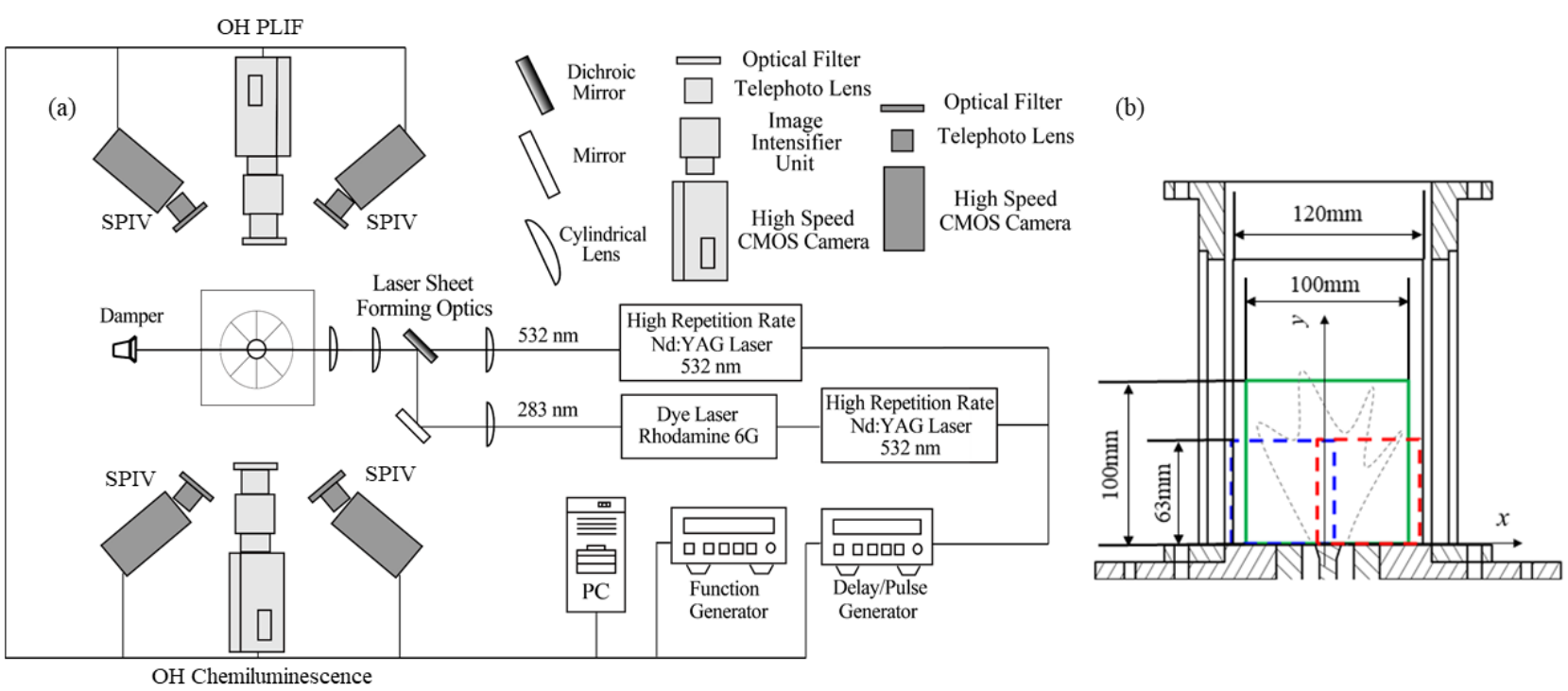

Fig. 2 (a) A schematic of setup of simultaneous measurements of SPIV and OH PLIF besides OH chemiluminescence. Two laser beams are led to the same axis and shaped into vertical laser sheets. Regarding SPIV, two sets of two CMOS cameras are used for multiple measurement regions side by side. (b) Measurement areas. Red and blue dashed squares of $63 \mathrm{~mm} \times 63 \mathrm{~mm}$ represent areas for SPIV, and the green solid square of $100 \mathrm{~mm} \times 100 \mathrm{~mm}$ shows an area both for chemiluminescence and PLIF.

combustor. Flames are anchored by the central recirculation zone. The swirl number $S$ at the inlet of the combustion chamber is calculated by

$$
S=\frac{\int_{R_{\text {in }}}^{R_{\text {out }}} v_{0} w_{0} x^{2} d x}{\left(R_{\text {out }}-R_{\text {in }}\right) \int_{R_{\text {in }}}^{R_{\text {out }}} v_{0}^{2} x d x}
$$

where $R_{\text {in }}, R_{\text {out }}, v_{0}, w_{0}$ are respectively semidiameters of the bluff-body and the inlet, axial and azimuthal velocities at the inlet. $S$ was calculated as 1.14 based on velocity distributions at $0.56 \mathrm{~mm}$ downstream of the inlet measured by SPIV operated under the stable state.

\subsection{Simultaneous measurement method of OH PLIF/Chemiluminescence and SPIV}

The schematic diagram of the experimental setup is shown in Fig. 2(a). The SPIV consists of a single pulsed Nd:YAG laser (Lee Laser LDP-100MQG, $532 \mathrm{~nm}, 2 \mathrm{~mJ} /$ pulse at $20 \mathrm{kHz}$ ), and four high-speed CMOS cameras (Photron, SA-Z, 1024 pixel×1024 pixels, $20 \mathrm{kHz}$ ) with lenses (AI AF Micro-Nikkor $200 \mathrm{~mm}$ f/4D IF-ED) and the optical band-pass filters (forward scattering: Edmund Optics, BANDPASS FILTER, CWL (Center Wavelength) 525 nm, FWHM (full width at the half maximum) $50 \mathrm{~nm}$; backward scattering: Semrock, FF01-525/15-50-D). The lenses are inclined 20 degrees with respect to the normal to a measurement plane, and the Scheimpflug condition (Prasad, 1995) was met. Spherical porous $\mathrm{SiO}_{2}$ particles of which mean particle diameter is $2 \mu \mathrm{m}$ (Suzukiyushi K.K, Godd Ball, E-6C) were used as tracer particles for SPIV. A PIV algorithm which has been developed in our previous studies (Tanahashi, et al., 2000, 2008; Shimura, et al., 2011; Naka, et al., 2016) was used to evaluate velocity from the successive particle images. 2D calibration (Prasad, 2000) was applied to each particle images. Instantaneous velocity was evaluated by 2-step hierarchical method (Kumar and Banerjee, 1998) and a window-offset method (Westerweel, et al., 1997). To eliminate spurious vectors, temporal and spatial Fourier cut-off filters (Tanahashi, et al., 2008) were applied.

As for OH PLIF measurement, the laser system consists of a Nd:YAG laser (Edgewave, IS120-2-L, $532 \mathrm{~nm}$, $5 \mathrm{~mJ} / \mathrm{pulse}$ ) and a dye laser (LIOP-TEC, LiopStar-HQ, Rhodamine 6G), and generates laser pulses of about $45 \mu \mathrm{J} /$ pulse. The dye laser with Rhodamine $6 \mathrm{G}$ dye in ethanol solvent is pumped by the Nd:YAG laser and emits 
Table 1 Measurement condition

\begin{tabular}{c|c|c|c}
\hline \hline & SPIV & Chem. \& PLIF & Pressure \\
\hline Field of view & $120 \mathrm{~mm} \times 63 \mathrm{~mm}$ & $100 \mathrm{~mm} \times 100 \mathrm{~mm}$ & \\
Spatial resolution & $1.10 \mathrm{~mm} \times 1.10 \mathrm{~mm}$ & $0.10 \mathrm{~mm} \times 0.10 \mathrm{~mm}$ & \\
Measurement repetition & $20 \mathrm{kHz}$ & $10 \mathrm{kHz}$ & $20 \mathrm{kHz}$ \\
\hline
\end{tabular}

approximately $283 \mathrm{~nm}$ light. The fluorescence from the A-X $(1,0)$ and $(0,0)$ bands $(306-320 \mathrm{~nm})$ was collected with a lens (UV-Nikkor $105 \mathrm{~mm} / \mathrm{F} 4.5$ ) through the optical band-pass filter (Semrock, FF01-320/40-50-D) and intensified by the image intensifier (Hamamatsu Photonics, C10880-03F, Gain 970, Gate $40 \mathrm{~ns}$ ), then, imaged onto a high-speed CMOS camera (Photron, SA-X2, 1024 pixel×1024 pixels, $10 \mathrm{kHz}$ ).

Regarding measurement of $\mathrm{OH}$ chemiluminescence, light emission was collected with a lens (UV-Nikkor $105 \mathrm{~mm} / \mathrm{F} 4.5$ ) through the optical band-pass filter (Semrock, FF01-320/40-50-D) and intensified by the image intensifier (Hamamatsu Photonics., C10880-03F, Gain 870, Gate $80 \mu$ s), then, imaged onto a high-speed CMOS camera (Photron, SA-X2, 1024 pixel $\times 1024$ pixels, $10 \mathrm{kHz}$ ).

Concerning measurement of pressure fluctuation, signals from high temperature tolerant sensor (Kistler, 7061C) are amplified (Kistler, Type 5018) and lead into A/D board (National Instruments, PCI-6115). The synchronous control system is composed by delay pulse generators (BNC Model 575, BNC Model 577, Stanford Research System DG645). As a combustion oscillation onset is nearly unpredictable under the experimental condition in this paper, pressure fluctuation is recorded ever since the flames are set to be the condition, and a trigger signal which is sent by a waveform generator (Keysight Technology, 33512B) to all of the cameras as a pre-trigger to record along the logs of pressure fluctuation.

\subsection{Measurement and Experimental condition}

Figure 2(b) shows measurement areas. Two dashed $63 \mathrm{~mm} \times 63 \mathrm{~mm}$-sized red and blue squares represent areas of SPIV which form a wide measurement area of velocity field. The $100 \mathrm{~mm} \times 100 \mathrm{~mm}$-sized green solid square is measurement area for PLIF and chemiluminescence, which is large enough to observe the whole flames even under the strong fluctuation so that $\mathrm{OH}$ chemiluminescence measurements have sufficient correlation with HRR (Röder, et al., 2012). Spatial resolution of SPIV based on size of the final interrogation window of 16 pixel $\times 16$ pixel was $1.1 \mathrm{~mm} \times 1.1 \mathrm{~mm}$. Overlap of the interrogation windows was $50 \%$ and therefore the vector spacing was $0.55 \mathrm{~mm}$. The spatial resolution of PLIF in the in-plane directions was $0.10 \mathrm{~mm} \times 0.10 \mathrm{~mm}$. The laser beam for SPIV was shaped into vertical sheet of $230 \mu \mathrm{m}$ FWHM thickness and $80 \mathrm{~mm}$ height, and that of PLIF was shaped into vertical sheet of $100 \mu \mathrm{m}$ FWHM thickness at the central axis of the chamber and $100 \mathrm{~mm}$ height. Measurement frequencies were 20 $\mathrm{kHz}$ for SPIV and pressure fluctuation, and $10 \mathrm{kHz}$ for PLIF and chemiluminescence. The measurement conditions are summarized in Table 1.

The experiments were performed under the condition of equivalence ratio of 0.69 and total flow rate of $350 \mathrm{~L} / \mathrm{min}$ where the transition process is observed. Although there also occurs the transition process under the sets of condition $(0.67,250 \mathrm{~L} / \mathrm{min})$ and $(0.65,200 \mathrm{~L} / \mathrm{min})$, they are much more difficult to measure since the transition process rarely happens.

\section{Results and discussions}

\subsection{Organization of physical properties fluctuation in the progress toward combustion oscillation}

Figure 3(a) shows a typical temporal evolution of pressure fluctuation $p$ ' including a transition process from stable to unstable of combustion oscillation. The transition process gets initiated at $t=0$. The magnitude of pressure fluctuation gradually increase as the transition process goes on. Figure 3(b) shows power spectra of pressure fluctuation during stable state and combustion oscillation state. The spectra show that both states have feature frequencies. Each dominant frequency $f_{\text {stable }}$ and $f_{\text {osc. }}$. was approximately $78 \mathrm{~Hz}$ and $88 \mathrm{~Hz}$ under the condition, which were slightly higher than the frequency of quarter-wave longitudinal acoustic mode of the combustion chamber at about $300 \mathrm{~K}$. According 

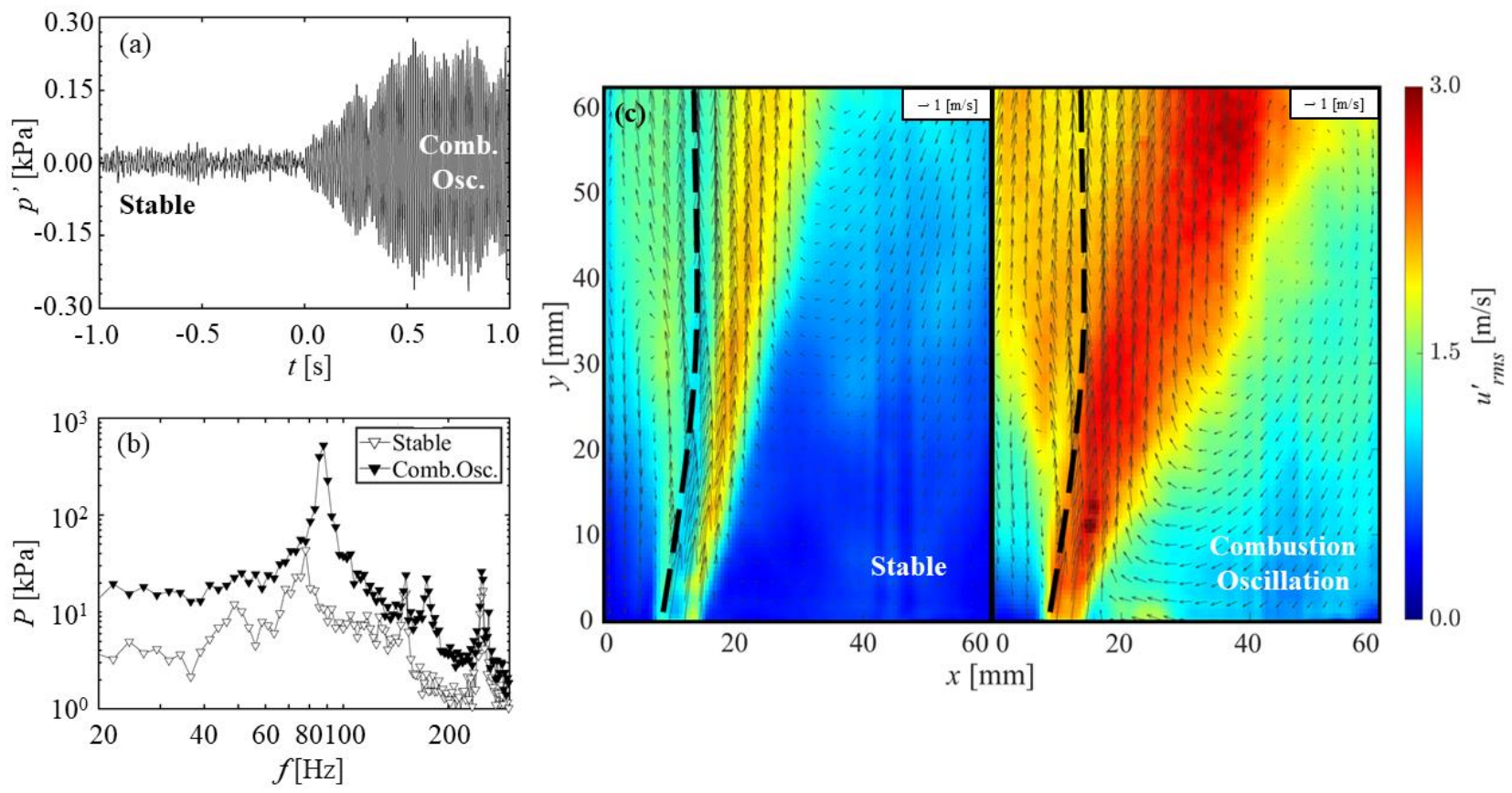

Fig. 3 (a) An example of time series of pressure fluctuation $p$ ' which includes the transition process from stable state to combustion oscillation, which shows gradual increase of its magnitude between those two states. (b) Averaged power spectra of pressure fluctuation in stable state and combustion oscillation. The dominant frequencies were approximately $78 \mathrm{~Hz}$ and $88 \mathrm{~Hz}$, respectively. The peak power became as high as 10 times. (c) A distribution of rms value of velocity fluctuation $u_{r m s}^{\prime}$ in those two states. The left half is for the stable states and the right one is for the combustion oscillation state. Dotted lines indicate the boundaries between the inside and outside of vorticity $\overline{\omega_{z}}=0$. The averaged in-plane velocity distributions were shown by sizes and directions of vectors

to Eq. 2, temperature distribution in the chamber is necessary for accurate prediction of dominant frequency. The reason of frequency differences would be explained by a temperature increase and a rise of $c$. Concerning magnitudes of spectra, peak power became as high as 10 times. There are also peaks of integer multiple of dominant frequencies, however the peaks around $150 \mathrm{~Hz}$ should be some noises as they appear when air does not flow. Figure 3(c) shows an averaged in-plane velocity distribution by sizes and directions of vectors and a velocity fluctuation distribution $u_{r m s}^{\prime}$ by overlapped colormap in those two states. $u^{\prime}{ }_{r m s}$ was calculated by

$$
u_{r m s}^{\prime}=\sqrt{\frac{\sum_{i=x, y, z} V_{i, r m s}^{\prime 2}}{3}}
$$

where $V_{i, r m s}^{\prime}$ are rms values of velocity fluctuation distribution in $i=x, y$ and $z$ direction. Dotted lines indicate the boundaries between the inside and outside of vorticity $\overline{\omega_{z}}=0$. In the transition process, the outer and inner recirculation zones (ORZ/IRZ) are difficult to be defined since the flow fluctuate irregularly. For organizing the characteristics in the IRZ, directions of rotation of inner plane velocity in the stable state are applied to represent circulation strength of each zone. The areas that have strong fluctuation apparently expand around the sheer layers.

In this study, the transition process was quantified corresponded to a time-series data of $p$ ' as follows:

1. Apply a band-pass filter to a time-series data of $p^{\prime}$. In this study, the targeted frequency ranges from $20 \mathrm{~Hz}$ to $300 \mathrm{~Hz}$. 



Fig. 4 (a) An example of temporal evolution of rms value of pressure fluctuations $p^{\prime}{ }^{\prime}$ 'ms calculated by using samples in every $0.1 \mathrm{sec}$ before and after each time. The vertical dashed lines show the time when $p^{\prime}{ }^{\prime}{ }^{\prime}$ s gains its intensity by $10 \%$ and $90 \%$ which are defined as the start $t=0$ and the end $t=T$ of the transition process towards combustion oscillation. The horizontal dashed lines indicate the mean $p^{\prime}{ }_{r m s}$ in the stable/unstable states, $p^{\prime}{ }_{\text {rms stable }}$ and $p_{\text {rms,osc. }}$. (b) Heat release changes $I^{*}$ estimated by $\mathrm{OH}$ chemiluminescence in the transition process. (c) An example of temporal evolution of fluctuation magnitudes of circulation to their sectional average $\left|\Gamma_{r m s} / \Gamma_{\text {ave }}\right|$ in the inside and outside of the boundary of vorticity $\overline{\omega_{z}}=0$.

2. Calculate the root-mean-square (rms) values $p^{\prime}{ }^{\prime}$ rms of $p^{\prime}$ using samples in every $0.1 \mathrm{sec}$ before and after each time.

3. Calculate the mean $p^{\prime}{ }_{r m s}$ in the stable/unstable states, $p_{r m s, s t a b l e}^{\prime}$ and $p_{r m s, o s c .}^{\prime}$. In this study, start of the transition process is defined as when $p^{\prime}{ }_{r m s}$ gains its intensity monotonously by $10 \%$ of $p^{\prime}{ }_{r m s, o s c}-p_{r m s, s t a b l e}$, and end of the transition process is done as when $p^{\prime}{ }^{\prime} r m$ gains by $90 \%$ as well. The time taken in the transition process is expressed as $T$.

Figure 4(a) indicates the time-series $p^{\prime}{ }_{r m s}$ of $p^{\prime}$ shown in Fig. 3(a). The $p^{\prime}{ }_{r m s, s t a b l e}$ and $p^{\prime}{ }_{r m s, o s c}$ are $0.0136 \mathrm{kPa}$ and $0.1446 \mathrm{kPa}$, which indicate that $p_{\text {rms }}^{\prime}$ gains almost 10 times as well as power peaks. In this study, the results of simultaneous measurements were organized to be averaged or integrated in accordance with the phases of pressure fluctuation and temporal sections in the transition process divided by 10 between $t=0$ and $t=T$. Figure 4 (b) and (c) indicate changes of sectional amount of heat release and circulation magnitudes $|\Gamma|$ in the inner zone and the outer zone of $\overline{\omega_{z}}=0$. The time-series of the amount of heat release $I^{*}$ is estimated by sectional integrated values of spatial averaged $\mathrm{OH}$ chemiluminescence distributed in green square in Fig. 2(b) and Fig. 5(a). The time-series change in Fig. 4(b) is expressed by proportions to the value integrated from $t=0$ to $t=T$. The figure shows that $I^{*}$ slightly elevates gradually, which means that the flame tends to become more compact in the transition process. Figure 4(a) and Fig. 4(b) indicates that magnitude of pressure fluctuation and HRR elevate simultaneously. The $\Gamma$ were calculated by the Stokes' theorem,

$$
\Gamma=\oint_{c} \boldsymbol{v} \cdot d \boldsymbol{l}=\int_{s} \boldsymbol{\omega} \cdot d \mathbf{s}
$$

where $c$ and $s$ indicate an arbitrary closed loop and areal elements in the enclosed region. The area was defined as the area surrounded by outer periphery of measurement area and the boundaries of $\overline{\omega_{z}}=0$ under the stable state and applied in common to the distributions in the transition process. The boundaries are shown by dotted lines in Fig. 3(c). As $\overline{\omega_{z}}$ has opposite sign of positive and negative across the central axis of the chamber, the circulation magnitudes were calculated in each side and evaluated by averaged absolute values. Fig. 4(c) also indicates gradual increase along the transition process, which indicates that the flame stabilizing effect of IRZ would come to collapse around $T / 2$.

\subsection{Flow characteristics and flame structures in the process}

Figure 5 shows a set of the simultaneous measurements at a time between $5 T / 10$ and $6 T / 10$. Red rectangular shows the same region in each measurement. Regarding the results of measurements of $\mathrm{OH}$ chemiluminescence, bright zones 

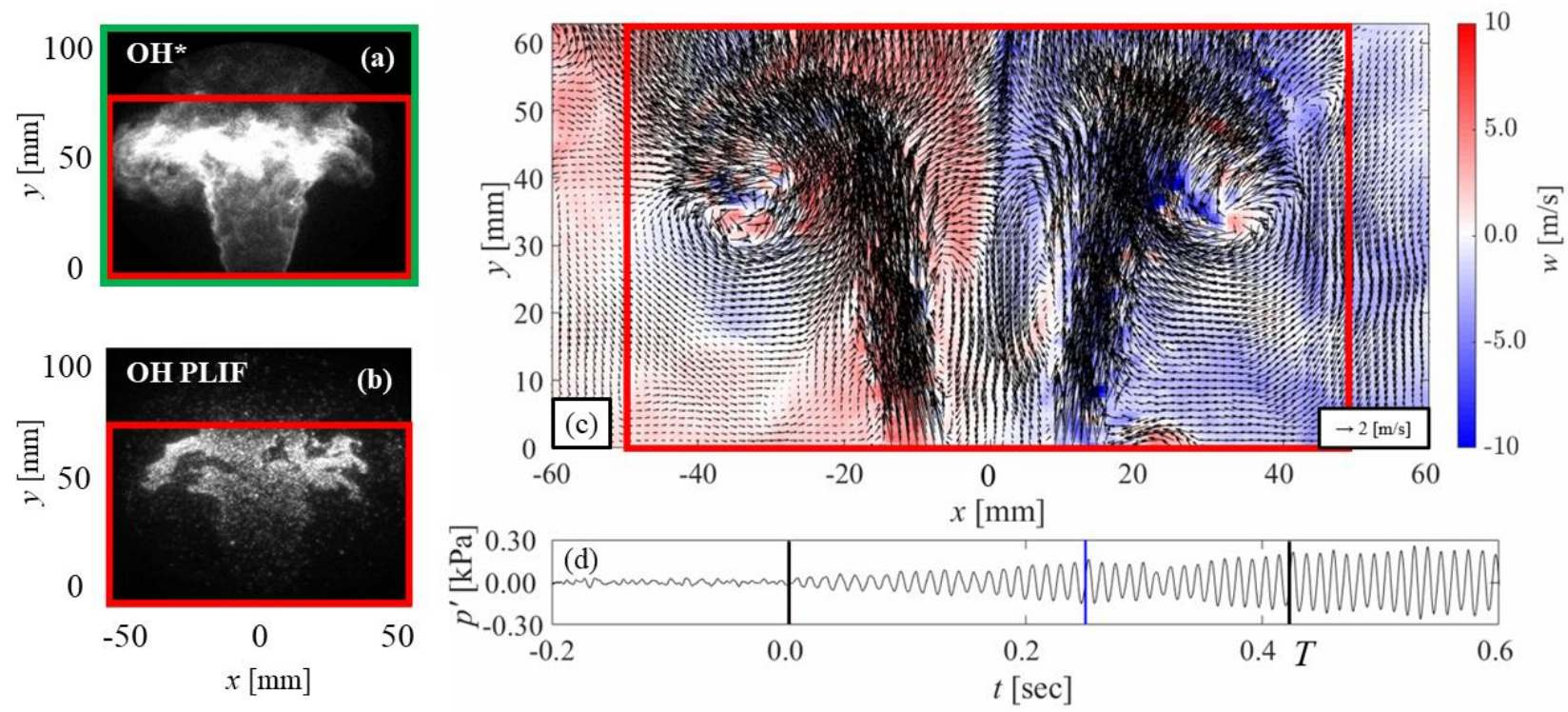

Fig. 5 An example of results of the simultaneous measurement for (a) OH chemiluminescence, (b) OH PLIF, (c) SPIV, (d) pressure fluctuation $p^{\prime}$. Each result is at the same time referred as blue line in Fig. 5(d). Red rectangular shows the same region in each measurement. Green rectangular matches with that in Fig. 2(b). $\mathrm{OH}$ chemiluminescence indicates integrated light emission in line of sight, whereas OH PLIF shows OH radical distribution in the cross section. The bright zones in both figures roughly show existences of large heat release and burnt region, respectively. In the result of SPIV, sizes and directions of vectors show in-plane velocity while out-of-plane velocity is shown by overlapped colormap.
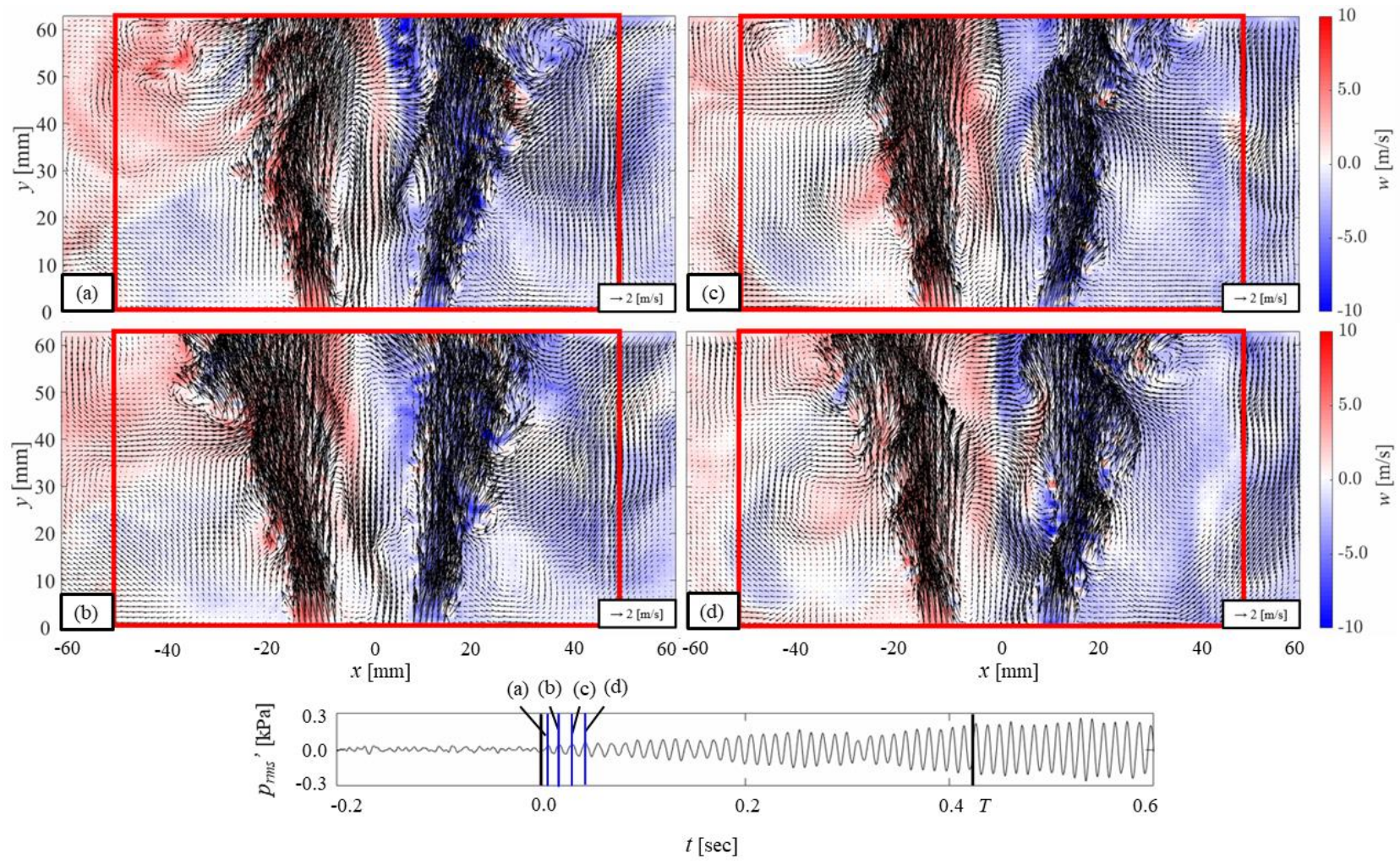

Fig. 6 Velocity distributions at the phase when pressure fluctuation takes its maximum. (a)-(d) corresponds to each time shown by the blue line in the (e) pressure fluctuation, which is right after starting the transition process. 

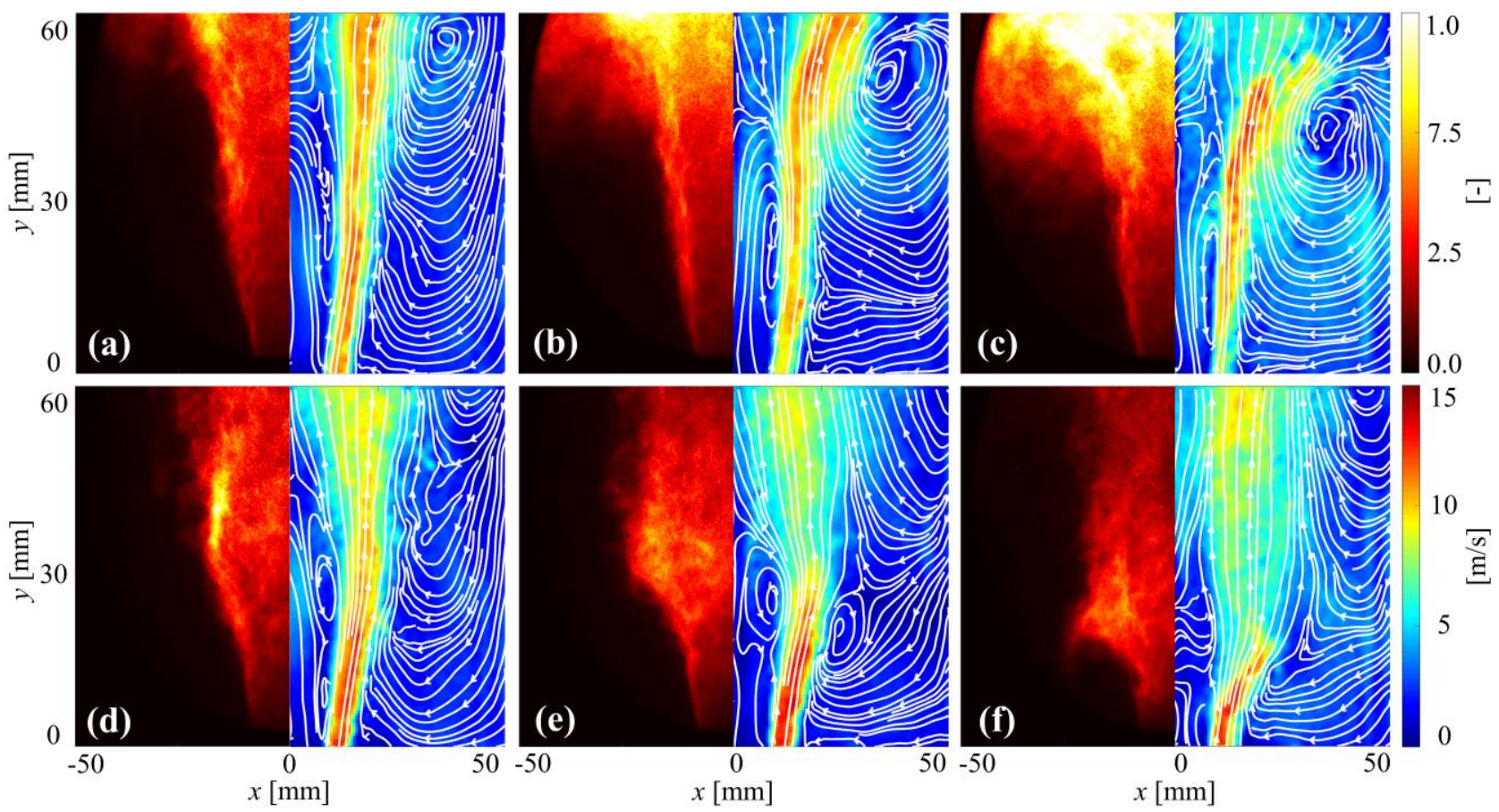

Fig. 7 Phase-averaged distributions of $\mathrm{OH}$ chemiluminescence (left half) and magnitude of streamwise velocity overlapped with streamlines (right half) corresponding to the phases of pressure fluctuation in (a)(d) $T / 10-2 T / 10$, (b)(e) 5T/10-6T/10, (c)(f) 9T/10-T. (a), (b) and (c) are the results when the pressure fluctuation takes its maximum in each section. On the other hand, (d), (e) and (f) are the results when the pressure fluctuation takes its minimum.

roughly show existences of high heat release region in line of sight (Ayoola, et al, 2006). As for OH PLIF, although the intensity of OH-PLIF is dependent on the intensity of the laser, it can predict burnt regions in premixed flames since $\mathrm{OH}$ radicals steeply increase around flame fronts. In the result of SPIV shown in Fig. 5(c), sizes and directions of vectors show in-plane velocity while out-of-plane velocity is shown by overlapped colormap. Each result is at the same time referred as blue line in time series of $p^{\prime}$, as shown in Fig. 5(d). This set corresponds to the result at the moment of high $\mathrm{d} p$ '/d $t$. In the result of chemiluminescence, large heat release region locates downstream side of large vortices. Out-of-plane velocity fluctuate in the large-scale vortices which indicates that some asymmetry features appear. Concerning the PLIF result, the vortical motion make burnt regions involve unburnt regions and form folding and peninsular structures. Although there is lack of the factors to be considered, the involving might boost the combustion in that remaining unburnt region changes into burnt region. Figure 5 shows the velocity distributions at first four times when pressure fluctuation takes its maximum. Observing the time series motion of velocity fields, there are asymmetrical vortex generations and the sheer layers are swinging. Those are gradually tuned towards the end of the transition process for combustion oscillation mode which is axial symmetry and axial back and forth movements as shown in Fig. 5(c).

Figure 7 shows phase averaged $\mathrm{OH}$ chemiluminescence and velocity fields corresponding to the phases of pressure fluctuation in (a)(d) $T / 10-2 T / 10$, (b)(e) 5T/10-6T/10, (c)(f) $9 T / 10-T$. The instantaneous results in each temporal section in each quartered phase of pressure fluctuation are averaged. Figure 7(a), (b) and (c) are the results in the phase of pressure fluctuation when it takes its maximum in each section. On the other hand, Fig. 7(d), (e) and (f) are the results in the phase of pressure fluctuation when it takes its minimum. The velocity fields show the in-plane streamlines and stream-wise speed distributions for overlapped colormap. Note that these are cross-sectional distributions which differs in the chemiluminescence ones. At the phase with the maximum of pressure fluctuation in each temporal section, the high HRR region and large-scale circulation can be found in the relatively downstream region of $y \approx 40-60 \mathrm{~mm}$, while in the phase of the minimum of pressure fluctuation, velocity near the inlet is enhanced and flame wrinkles around $y \approx 15-30 \mathrm{~mm}$. These characteristics of phase averaged velocity coincide well with the results in the previous study 

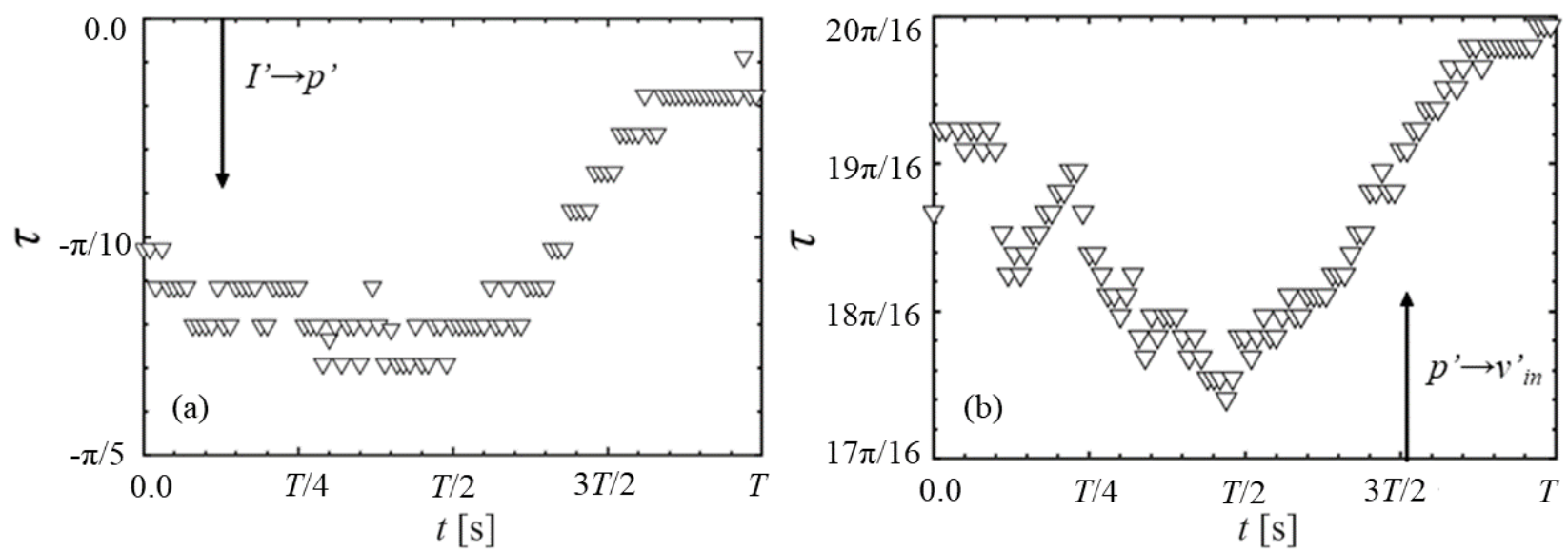

Fig. 8 Temporal evolution of phase difference $\tau$ between (a) pressure fluctuation $p$ ' and fluctuation of $\mathrm{OH}$ chemiluminescence intensity $I^{\prime}$ in the transition process and between (b) $p$ ' and in-flow velocity fluctuation $v^{\prime}{ }_{i n} . I^{\prime}$ is calculated by spatial averaged $\mathrm{OH}$ chemiluminescence. $\tau$ was calculated based on the frequency of combustion oscillation. Direction of each arrow shows that phases of $p$ ' get behind by $I^{\prime}$ while $p$ ' gets forward by $v^{\prime}$ in.

(Shimura, et al., 2009). It is worth looking that the large heat release regions gradually expand with the increment of the temporal section, and edges of the regions make large curve to be adjacent to the high-speed regions. The center of vortices tends to move in the upstream direction. The temporal change of dominant frequency is difficult to get since the transition process is ms order of very short. On the other hand, the position of the large-scale vortices would have some effect as shortening the length of $n_{x}$ by $10 \mathrm{~mm}$ leads to lower the dominant frequency by several $\mathrm{Hz}$ according to Eq. 2. Moreover, the gradual increase of $I^{*}$ in the Fig. 4(b) would be explained by a movement of flame position to the integrated area, that is, flames also tends to move in the upstream direction. Furthermore, Fig. 7(f) shows collapse of flow structures on the top of bluff-body apparently, which corresponds to Fig. 4(c). When it comes to look at the velocity magnitude right after the inlet, the magnitude is reduced gradually when the pressure fluctuation takes its maximum, which means that the velocity of the inlet flow comes to fluctuate gradually with tuning to the phase of pressure fluctuation and HRR.

Figure 8(a) shows temporal evolution of phase difference $\tau$ between fluctuations of pressure and $\mathrm{OH}$ chemiluminescence intensity $I^{\prime}$ which is specified as the gap at which the cross-correlation coefficient become maximum. $I^{\prime}$ is calculated by spatial average of $\mathrm{OH}$ chemiluminescence. Cross correlation was calculated by using samples in $0.1 \mathrm{sec}$ before and after each sampling time. $\tau$ was calculated based on the frequency of combustion oscillation. According to Fig. 8(a), phase difference between $p$ ' and I' started from about $-\pi / 8$ and became smaller as the transition process advanced. The difference is always small enough to hold good for Rayleigh criteria shown in Eq. (1). Figure 8(b) shows the temporal evolution of $\tau$ between fluctuations of pressure and magnitude of in-flow velocity $v_{\text {in. }}^{\prime}$. Different from the relation between $p$ ' and $I^{\prime}$, phase differences between $p$ ' and in-flow velocity fluctuation $v^{\prime}$ in were approximately antiphase, which seems to be connected with the relation between static pressure and dynamic pressure. However, the phase difference indicates a specific delay of about $5 \pi / 4$ or $-3 \pi / 4$ at combustion oscillation state. Negative delay here means that the in-flow velocity fluctuation is in advance. At around $t=T / 2$, the delay was getting close to $\pi$. There is a possibility that this phase change would have effects on the slight decrease of pressure fluctuation at around $0.3 \mathrm{~s}$ in Fig. 5(d).

\section{Summary and conclusion}

In this study, turbulent swirling lean premixed flames in a transition process of combustion oscillation in a swirl premixed combustor were investigated to clarify the dynamical characteristics of the combustion oscillation onset. The operational condition for the experiment was set as equivalence ratio of 0.69 , total flow rate of $350 \mathrm{~L} / \mathrm{min}$. The transition process was defined based on the time-series rms values of pressure fluctuation, which enabled to quantify some synchronized elevating properties. Forming of large vortical motion, heat release and pressure fluctuation are 
mutually affected, especially the vortexes gradually moved to the inlet-wise and the flames are severely distorted. The periodical relation is tuned complicatedly step by step in the transition process. Phase difference between fluctuations of pressure and heat release got close to zero and that between fluctuations of pressure and magnitude of inflow velocity got close to $5 \pi / 4$ in the combustor. The temporal change in phase difference $\tau$ between $p$ 'and $v^{\prime}$ in would be one of control methods in addition to the that between $p$ 'and $I$ '.

\section{Acknowledgment}

This research is partially granted by the Japan Society for the Promotion of Science (JSPS) through No. $16 \mathrm{H} 06069$ and $18 \mathrm{KK} 0400$.

\section{References}

Aoki, K., Shimura, M., Ogawa, S., Fukushima, N., Naka, Y., Nada, Y., Tanahashi, M. and Miyauchi, T., Short- and long-term dynamic modes of turbulent swirling premixed flame in a cuboid combustor, Proceedings of the Combustion Institute, Vol.35, No.3 (2015), pp.3209-3217.

Ayoola, B. O., Balachandran, R., Frank, J. H., Mastorakos, E. and Kaminski, C. F., Spatially resolved heat release rate measurements in turbulent premixed flames, Combustion and Flame, Vol.144, No.1-2 (2006), pp.1-16.

Bomberg, S., Emmert, T. and Polifke, W., Thermal versus acoustic response of velocity sensitive premixed flames. Proceedings of the Combustion Institute, Vol.35, No.3 (2015), pp.3185-3192.

Boxx, I., Slabaugh, C., Kutne, P., Lucht, R. P. and Meier, W., 3 kHz PIV/OHPLIF measurements in a gas turbine combustor at elevated pressure, Proceedings of the Combustion Institute, Vol.35, No.3 (2015), pp.3793-3802.

Boxx, I., Stöhr, M., Carter, C. and Meier, W., Temporally resolved planar measurements of transient phenomena in a partially pre-mixed swirl flame in a gas turbine model combustor, Combustion and Flame, Vol.157, No.8 (2009), pp.1510-1525.

Candel, S., Combustion dynamics and control: Progress and challenges, Proceedings of the Combustion Institute, Vol.29, No.1 (2002), pp.1-28.

Choi, G.-M., Tanahashi, M., and Miyauchi, T., Control of oscillating combustion and noise based on local flame structure, Proceedings of the Combustion Institute, Vol.30, No.2 (2005), pp.1807-1814.

Huang, Y. and Yang, V., Bifurcation of flame structure in a lean-premixed swirl-stabilized combustor: Transition from stable to unstable flame, Combustion and Flame, Vol.136, No.3 (2004), pp.383-389.

Huang, Y. and Yang, V., Dynamics and stability of lean-premixed swirl-stabilized combustion, Progress in Energy and Combustion Science, Vol.35, No.4 (2009), pp.293-364.

Kumar, S. and Banerjee, S., Development and application of a hierarchical system for digital particle image velocimetry to free-surface turbulence, Physics of Fluids, Vol.10 (1998), pp.160-177.

Li, Y., Tanahashi, M. and Miyauchi, T., Sound generation in chemically reacting mixing layers, Transactions of the Japan Society of Mechanical Engineers, Series B, Vol.66, No.648 (2000), pp.2117-2124.

Lieuwen, T., Modeling Premixed combustion-acoustic wave interactions: A Review, Journal of Propulsion and Power, Vol.19, No.5 (2003), pp.765-781.

Nair, V., Thampi, G., Sujith, R. I., Intermittency route to thermoacoustic instability in turbulent combustors, Journal of Fluid Mechanics, Vol. 756 (2014), pp. 470-487.

Naka, Y., Tomita, K., Shimura, M., Fukushima, N., Tanahashi, M. and Miyauchi, T., Quad-plane stereoscopic PIV for fine-scale structure measurements in turbulence, Experiments in Fluids, Vol.57, No.63 (2016), 20pp.

Oberleithner, K., Schimek, S. and Paschereit, C. O., Shear flow instabilities in swirl-stabilized combustors and their impact on the amplitude dependent flame response: A linear stability analysis, Combustion and Flame, Vol.162, No.1 (2015), pp.86-99.

Poinsot, T., Prediction and control of combustion instabilities in real engines, Proceedings of the Combustion Institute, Vol.36, No.1 (2017), pp.1-28.

Prasad, A. K., Scheimpflug stereocamera for particle image velocimetry in liquid flows, Applied Optics, Vol.34, No.30 (1995), pp7092-7099.

Prasad, A. K., Stereoscopic particle image velocimetry, Experiments in Fluids, Vol.29 (2000), pp.103-116. 
Rayleigh, J., The Theory of Sound, (1878 reeditted in 1945), New York, Dover.

Renaud, A., Ducruix, S., Scouflaire, P., Zimmer, L., Flame shape transition in a swirl stabilised liquid fueled burner, Proceedings of the Combustion Institute, Vol. 35, No. 3 (2015), pp. 3365-3372.

Röder, M., Dreier, T. and Schulz, C., Simultaneous measurement of localized heat release with OH/CH${ }_{2} \mathrm{O}-\mathrm{LIF}_{\mathrm{imaging}}$ and spatially integrated $\mathrm{OH}^{*}$ chemiluminescence in turbulent swirl flames, Applied Physics B: Lasers and Optics, Vol.107, No.3 (2012), pp.611-617.

Shimura, M., Tanahashi, M., Choi, G. M. and Miyauchi, T., Large-scale vortical motion and pressure fluctuation in noise-controlled, swirl-stabilized combustor, Journal of Thermal Science and Technology, Vol.4, No.4 (2009), DOI: $10.1299 /$ jtst.4.494

Shimura, M., Ueda, T., Choi, G.-M., Tanahashi, M. and Miyauchi, T., Simultaneous Dual-Plane CH PLIF, Single-Plane $\mathrm{OH}$ PLIF and Dual-Plane Stereoscopic PIV Measurements in Methane-Air Turbulent Premixed Flames, Proceedings of the Combustion Institute, Vol.33 (2011), pp.775-782.

Shanbhogue, S., Shin, D. H., Hemchandra, S., Plaks, D. and Lieuwen, T., Flame sheet dynamics of bluff-body stabilized flames during longitudinal acoustic forcing, Proceedings of the Combustion Institute, Vol.32, No.2 (2009), pp.1787-1794.

Shroll, A. P., Shanbhogue, S. J., Ghoniem, A. F., Dynamic-stability characeristics of premixed methane oxy-combustion, Journal of Engineering for Gas Turbines and Power, Vol. 134, No. 5 (2012) pp. 470-487.

Steinberg, A. M., Arndt, C. M. and Meier, W., Parametric study of vortex structures and their dynamics in swirl-stabilized combustion, Proceedings of the Combustion Institute, Vol.34, No.2 (2013), pp.3117-3125.

Stöhr, M., Sadanandan, R. and Meier, W., Experimental study of unsteady flame structures of an oscillating swirl flame in a gas turbine model combustor, Proceedings of the Combustion Institute, Vol.32, No.2 (2009), pp.2925-2932.

Tanahashi, M., Ootsu, M., Fukushima, M. and Miyauchi, T., Measurement of coherent fine scale eddies in turbulent mixing layer by DPIV, Engineering Turbulence Modelling and Experiments Vol.5 (2002), pp.525-534.

Tanahashi, M., Hirayama, T., Taka, S. and Miyauchi, T., Measurement of fine scale structure in turbulence by time-resolved dual-plane stereoscopic PIV, International Journal of Heat and Fluid Flow, Vol.29 (2008), pp.792-802.

Tanahashi, M., Shimura, M. and Minamoto, Y., Progress in DNS and laser diagnostics of turbulence and turbulent combustion, Thermal Science and Engineering, Vol.25, No.3 (2017), pp.27-43.

Westerweel, J., Dabiri, D. and Garib, M., The effect of a discrete window offset on the accuracy of cross-correlation analysis of digital PIV recordings, Experiments in fluids, Vol.23 (1997), pp.20-28.

Weigand, P., Meier, W., Duan, X. R., Stricker, W. and Aigner, M., Investigations of swirl flames in a gas turbine model combustor: I. Flow field, structures, temperature, and species distributions, Combustion and Flame, Vol.144, No.1-2 (2006), pp.205-224. 